\title{
Photocycle features of heterologously expressed and assembled eukaryotic flavin-binding BLUF domains of photoactivated adenylyl cyclase (PAC), a blue-light receptor in Euglena gracilis
}

\author{
Shinji Ito, ${ }^{a, b}$ Akio Murakami, ${ }^{c}$ Kyosuke Sato, ${ }^{d}$ Yasuzo Nishina, ${ }^{d}$ Kiyoshi Shiga, ${ }^{d}$ \\ Tetsuo Takahashi, ${ }^{e}$ Shoichi Higashi, ${ }^{b}$ Mineo Iseki ${ }^{b, f}$ and Masakatsu Watanabe ${ }^{* a, b}$ \\ ${ }^{a}$ Department of Photoscience, School of Advanced Sciences, Graduate University for Advanced \\ Studies (SOKENDAI), Shonan Village, Hayama, Kanagawa 240-0193, Japan. \\ E-mail: watanabe_masakatsu@soken.ac.jp; Fax:+81-46-858-1544; Tel: +81-46-858-1561 \\ ${ }^{b}$ National Institute for Basic Biology, National Institutes of Natural Sciences, Okazaki, \\ Aichi 444-8585, Japan \\ ${ }^{c}$ Kobe University Research Center for Inland Seas, Iwaya, Awaji, Hyogo 656-2401, Japan \\ ${ }^{d}$ Department of Molecular Physiology, Graduate School of Medical Sciences, Kumamoto \\ University, Honjo, Kumamoto, Kumamoto, 860-8556, Japan \\ e School of Pharmaceutical Sciences, Toho University, Miyama, Funabashi, Chiba 274-8510, \\ Japan \\ ${ }^{f}$ PRESTO, Japan Science and Technology Agency, Honcho, Kawaguchi, Saitama 332-0012, \\ Japan
}

Received 26th April 2005, Accepted 19th July 2005

First published as an Advance Article on the web 8th August 2005

Photoactivated adenylyl cyclase (PAC) is a recently discovered blue-light photoreceptor that mediates photomovement in Euglena gracilis (Iseki et al., Nature, 2002, 415, 1047-1051). PAC appears to be a heterotetramer composed of two FAD-binding subunits (PAC $\alpha$ and $\mathrm{PAC} \beta$ ). Both subunits have a pair of homologous regions (F1 and F2) which show homology with prokaryotic "sensors of blue-light using FAD" (BLUF) domains. The F1 and F2 domains of PAC are the only eukaryotic BLUF domains found thus far. We obtained soluble recombinant F1 and F2 proteins in PAC $\alpha$ by heterologous expression with fused glutathione- $S$-transferase (GST) in $E$. coli. The expressed F1 samples did not bind flavins, but the F2 samples contained both FAD and FMN with trace amounts of riboflavin. We also assembled the histidine-tagged recombinant F2 (6His-F2) from inclusion bodies in E. coli with exogenous FAD or FMN. Blue-light-induced changes in absorption spectra of these assembled samples were highly similar to those reported for prokaryotic BLUF domains. The FAD- or FMN-assembled 6His-F2 photocycled with nearly the same rate constants of light-reaction and dark-relaxation, which were slightly lower than those of GST-cleaved F2. The estimated quantum efficiency for the phototransformation was $0.28-0.32$, and the half-life was $34-44 \mathrm{~s}$ at $25^{\circ} \mathrm{C}$ for the recombinant PAC $\alpha$ F2, whereas that reported for prokaryotic BLUF domains varied from $c a .3 .5 \mathrm{~s}$ (T110078) to ca. $900 \mathrm{~s} \mathrm{(AppA).} \mathrm{The} \mathrm{mutated} \mathrm{recombinant} \mathrm{Y472F} \mathrm{and} \mathrm{Q514G} \mathrm{of} \mathrm{PAC} \alpha$ F2 and the F2 domain of the PAC $\alpha$ homologue from Eutreptiella gymnastica, which lacks the Gln residue conserved in other BLUF domains, showed no photoinduced transformation.

\section{Introduction}

The photosynthetic flagellate Euglena gracilis uses flagellarmediated photomovements to locate optimal light conditions for photosynthesis. Photoactivated adenylyl cyclase (PAC), which acts as a blue light regulated adenylyl cyclase, has been previously extracted from the paraflagellar bodies (PFBs) near the base of the flagella, and identified as the blue-light photoreceptor which mediates the step-up photophobic response (turning of the cell in response to abrupt increases in light intensity), resulting in avoidance from intense illumination. ${ }^{1,2}$ PAC noncovalently binds flavin adenine dinucleotides (FAD), and is composed of two subunits (PAC $\alpha$ and PAC $\beta$ ) which are similar to each other. Both subunits have a pair of homologous regions ( $\mathrm{F} 1$ and $\mathrm{F} 2$ ) showing also homology with prokaryotic BLUF domains. ${ }^{3}$ F1 and/or F2 appear to be FAD-binding domains which sense blue light using FAD. It is possible that a photosignal captured in F1 and/or F2 is transferred to a catalytic domain (probably $\mathrm{C} 1$ and/or $\mathrm{C} 2$ ) through a conformational change, consequently elevating adenylyl cyclase activity. Other euglenoids have orthologues of PAC $\alpha$ and PAC $\beta$ with homologous domains of F1 and F2. ${ }^{4}$ These BLUF homologues constitute the only cases found so far in eukaryotic organisms.

AppA, which has a single BLUF domain, ${ }^{5}$ is another blue-light photoreceptor that functions as an antirepressor of photosynthetic gene expression in the purple bacterium Rhodobacter sphaeroides. ${ }^{6,8}$ A recombinant BLUF domain of AppA produced by expression in Escherichia coli displays a photocycle, formation of a signaling state by irradiation of blue light followed by dark regeneration of its original state, resulting in a red-shift of the absorption spectrum. ${ }^{6-13}$ Slr1694, which has a single BLUF domain, is probably involved in positive phototaxis in Synechocystis sp. PCC 6803. ${ }^{14}$ This recombinant BLUF domain shows a similar photocycle to that of AppA. ${ }^{15}$ Recently, ultrafast spectroscopic analysis of the recombinant BLUF domain of AppA and another cyanobacterial BLUF domain (Tl10078) showed that the signaling state is formed from the light-excited state within $10 \mathrm{~ns} .{ }^{13,16}$

Site-directed mutagenesis in the BLUF domains of AppA and Slr1694 suggests that a tyrosine residue conserved in all BLUF domains (at the 21 st position in AppA or at the 8th position in Slr1694) is involved in the photocycle, ${ }^{7,9,17}$ either through 
$\pi-\pi$ stacking interaction with the isoalloxazine ring of $\mathrm{FAD}^{7}$ or through proton transfer from FAD. ${ }^{9,10}$ Spectrochemical analysis revealed that light excitation leads to structural rearrangements of the protein moieties coupled with conformational changes of the isoalloxazine rings II and III of FAD, due to hydrogen bonding. ${ }^{12,15,17,18}$ The recently determined crystal structures of BLUF domains of AppA and T110078 showed that the Tyr21 in AppA and Tyr8 in T110078 are hydrogen bound to Gln63 and Gln50, respectively, and each of them directly interacts with FAD by forming a hydrogen bond with $\mathrm{O} 4$ of isoalloxazine ring III of FAD. ${ }^{19,20}$ Replacement of Gln50 with Ala in T110078 has the same effect as the Y8F mutant, i.e., suppression of the lightinduced absorption changes. ${ }^{19}$

The mechanism of photoactivation of PAC is of great interest because of the regulatory interaction of the flavin binding domain with the adenylyl cyclase catalytic domain. Characterization of the putative photocycle during the photochemical reaction of PAC should provide important information about the mechanism. However, it has been difficult to obtain enough amounts of PAC or its BLUF domains (F1 and/or F2) to study their photocycle spectrophotometrically, either by large-scale purification from PFBs or by heterologous expression. During the experiments reported in this paper we obtained sufficient amounts of soluble recombinant $\mathrm{PAC} \alpha \mathrm{F} 1$ and $\mathrm{F} 2$ proteins fused with glutathione- $S$-transferase (GST) by heterologous expression in E. coli. The F2 samples contained both FAD and FMN with trace amounts of riboflavin. ${ }^{21}$ Although it is assumed that native PAC $\alpha$ F1 binds FAD, the expressed F1 samples did not bind flavins. In addition, we developed a method for assembly of His-tagged recombinant PAC $\alpha$ F2 heterologously expressed in $E$. coli and obtained from inclusion bodies. Taking advantage of these recombinant $\mathrm{F} 2$ samples, we examined the basic photocycling characteristics using blue LED or $\mathrm{HeCd}$ laser excitation and dual-wavelength spectrophotometry. Here we discuss the photocycling characteristics of a recombinant eukaryotic BLUF domain, $\mathrm{PAC} \alpha \mathrm{F} 2$, as compared to prokaryotic BLUF domains.

Because Tyr472 and Gln514 in PAC $\alpha$ F2 correspond to Tyr21 in AppA or Tyr8 in Slr1694, and Gln63 in AppA or Gln50 in Tl10078, required for the photocycle, ${ }^{7,9,17,19,20}$ we made pointmutated recombinants (Y472F and Q514G mutants) proteins fused with GST. Furthermore, the F2 domain of the PAC $\alpha$ homologue of Eutreptiella gymnastica (EtgPAC1) ${ }^{4}$ lacks the Gln522 residue conserved in other PAC homologues and other BLUF domains. We heterologously expressed EtgPAC1 in $E$. coli and the above described three samples were used to examine whether the Tyr and the Gln residues in eukaryotic BLUF domains are essential for the photocycle, as in the prokaryotic BLUF domains.

\section{Materials and methods}

\section{Construction of expression vectors}

Recombinant PACa F2 was overexpressed as an N-terminal fusion with GST in $E$. coli using a tac promoter-based expression system, as shown in Fig. 1. To construct the expression plasmid, the region from Gly462 to Asp574 of PAC $\alpha$, which includes the F2 domain, was amplified using the polymerase chain reaction (PCR) with a DNA polymerase (Pyrobest, Takara, Ohtsu, Japan). Forward and reverse primers, 5'-ATGGGATCCGGTGGTGAAGGCCAG-3' and 5'-CGTCGACTAGTCCTGCAGGGGTGG-3', were used to amplify the F2 coding sequence, adding BamHI and SalI restriction sites and a stop codon. A cloned cDNA encoding PAC $\alpha$ was used as a PCR template. The PCR-amplified fragment was cloned into BamHI-SalI restriction sites of pGEX-6P2 (Amersham Bioscience, Piscataway, NJ, USA), resulting in recombinant plasmid pGEX-F2 for expression of the GST-fused F2 protein (GST-F2).

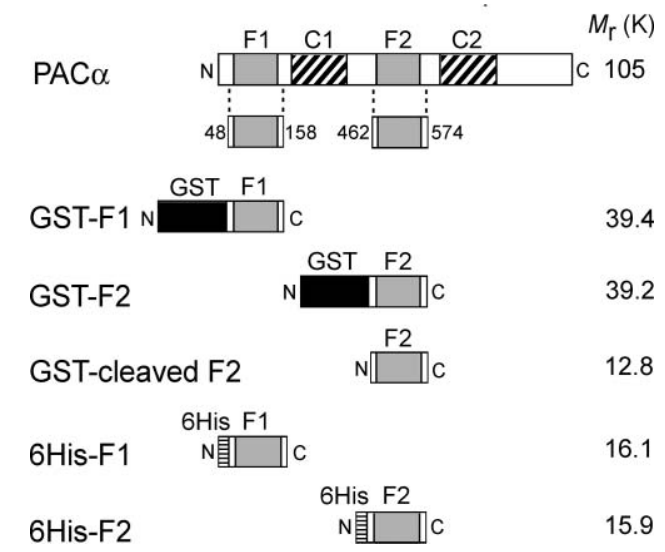

Fig. 1 Schematic structures of native PAC $\alpha$ and recombinant PAC $\alpha$ F1 and F2. Relative molecular masses $\left(M_{\mathrm{r}}\right)$ of GST-F1, GST-F2, GST-cleaved F2, 6His-F1, and 6His-F2 were calculated with the PeptideSort in GCG Wisconsin Package (Accelrys, San Diego, USA).

To construct the expression plasmids for the recombinant Y472F and Q514G mutants of PAC $\alpha$ F2, pGEX-F2 vectors were point-mutated by site-directed mutagenesis (QuickChange site-directed mutagenesis kit, Stratagene, La Jolla, CA, USA), resulting in pGEX-F2(Y472F) and pGEX-F2(Q514G) for expressions of point-mutated GST-F2 proteins [GST-F2(Y472F) and GST-F2(Q514G)], respectively.

Another construct for expression of recombinant PAC $\alpha$ F2 was made using a T7 RNA polymerase-based overexpression system that added a His-tag to the amino terminus of the protein. A DNA fragment including the multiple cloning site from plasmid pET28a(+) (EMD Biosciences, Darmstadt, Germany) was removed using ApaI and $X h o \mathrm{I}$ and was cloned into the ApaI and XhoI restriction sites of pET21d(+) (EMD Biosciences) for selection of transformed cells with ampicillin, resulting in plasmid pET21d(28a). Next, the PCR-amplified fragment of F2 was inserted into the multiple cloning site (using BamHISalI restriction sites) of pET21d(28a), resulting in recombinant plasmid pET21d(28a)-F2 for expression of the His-tagged F2 protein (6His-F2) (Fig. 1).

To construct the expression plasmids of PAC $\alpha \mathrm{F} 1$, the region from Gly48 to His158, which includes the F1 domain, was amplified using the PCR with DNA polymerase (Pyrobest, Takara) and the cloned cDNA encoding PAC $\alpha$. The PCR-amplified fragments of F1 were cloned into BamHI-SalI restriction sites of pGEX-6P-2 and pET21d(28a), resulting in recombinant plasmids pGEX-F1 and pET21d(28a)-F1 for expressions of the GST-fused F1 protein (GST-F1) and His-tagged F1 protein (6His-F1) (Fig. 1), respectively.

Recombinant EtgPAC1 F2 was overexpressed in E. coli using the T7 RNA polymerase-based expression system. The region from Met467 to Thr570 of EtgPAC1, which includes the EtgPAC1 F2 domain, was amplified by PCR using DNA polymerase (Pyrobest, Takara). Forward and reverse primers, 5'GGGAATTACATATGGCCGGAGGAGGC-3' and 5'-CGTCGACTACGTAGCAGCAGTCACAGAGA-3', were used to amplify the EtgPAC1 F2 coding sequence, adding NdeI and SalI restriction sites and a stop codon. A cloned cDNA encoding EtgPAC1 was used as a PCR template. The PCR-amplified fragment was inserted into NdeI-SalI restriction sites of pET21d(28a), resulting in recombinant plasmid pET21d(28a)-(EtgPAC1)F2 for expression of the His-tagged EtgPAC1 F2 protein (6His(EtgPAC1)F2).

\section{Expression and purification of GST-F1, GST-F2, GST-F2(Y472F), and GST-F2(Q514G)}

The recombinant plasmids pGEX-F1, pGEX-F2, pGEXF2(Y472F), and pGEX-F2(Q514G) were separately introduced into $E$. coli strain BL21 (Amersham Bioscience). The cells 
were cultured in Luria broth (LB) containing $100 \mu \mathrm{g} \mathrm{m} \mathrm{m}^{-1}$ ampicillin. GST-F2 was overexpressed by induction with $5 \mu \mathrm{M}$ isopropyl- $\beta$-D-thiogalactopyranoside (IPTG) at $15{ }^{\circ} \mathrm{C}$ for $15 \mathrm{~h}$ in darkness. Harvested cells were suspended in phosphate buffered saline, $\mathrm{pH} 7.4$, and broken by ultrasonication $(20 \mathrm{kHz}$, $50 \mathrm{~W}$ ). The soluble GST-F1, GST-F2, GST-F2(Y472F), and GST-F2(Q514G) in the supernatants after centrifugation (at $10000 \mathrm{~g}$ for $30 \mathrm{~min}$ at $4{ }^{\circ} \mathrm{C}$ ) of the sonicates were affinitypurified using GST-binding resin (Glutathione Sepharose 4B, Amersham Bioscience) and elution buffer, $50 \mathrm{mM}$ Tris- $\mathrm{HCl}$, containing $10 \mathrm{mM}$ reduced glutathione, $\mathrm{pH} 9$, as described by the manufacturer.

\section{Purification of GST-cleaved F2}

The above described GST-F2 bound to the GST-binding resin was cleaved using a protease (PreScission Protease, Amersham Bioscience) in a cleavage buffer as described by the manufacturer, and the soluble GST-cleaved F2 was then collected as supernatant after centrifugation (at $500 \mathrm{~g}$ for $10 \mathrm{~min}$ at $4{ }^{\circ} \mathrm{C}$ ).

\section{Expressions, solubilizations, and assemblies of 6His-F1 and 6His-F2}

The recombinant plasmids pET21d(28a)-F1 and pET21d(28a)F2 were introduced into E. coli strain BL21(DE3) (EMD Biosciences) and cultured as described above. The cells were cultured in LB containing $100 \mu \mathrm{g} \mathrm{ml}^{-1}$ ampicillin. $6 \mathrm{His}-\mathrm{F} 1$ and $6 \mathrm{His}-\mathrm{F} 2$ were overexpressed by induction with $0.5 \mathrm{mM}$ IPTG at $37{ }^{\circ} \mathrm{C}$ for $6 \mathrm{~h}$. Almost all of the expressed 6His-F1 and $6 \mathrm{His}-$ F2 were present in insoluble inclusion bodies. Harvested cells were suspended in potassium phosphate buffer, $\mathrm{pH}$ 7. The cells were broken by ultrasonication. Inclusion bodies were separated from the sonicate by centrifugation at $10000 \mathrm{~g}$ for $30 \mathrm{~min}$ at $4{ }^{\circ} \mathrm{C}$, and the pellet was resuspended and washed with $50 \mathrm{mM}$ potassium phosphate buffer, $\mathrm{pH} 7$, containing $4 \%$ Triton X-100, and finally suspended in $50 \mathrm{mM}$ potassium phosphate buffer, $\mathrm{pH}$ 7. An aliquot of the inclusion body suspension, containing $4.2 \mathrm{mg} \mathrm{ml}^{-1}$ total protein for $6 \mathrm{His}-\mathrm{F} 2$, was mixed with 3 parts of $8 \mathrm{M}$ guanidine- $\mathrm{HCl}$ solution, resulting in a denatured protein solution.

Protein-refolding of $6 \mathrm{His}-\mathrm{F} 2$ was performed by rapid manual mixing of an aliquot of the suspension of denatured 6HisF2 solution with 12.5 parts of the refolding buffer: $50 \mathrm{mM}$ potassium phosphate buffer, $\mathrm{pH} 7$, containing $20 \mu \mathrm{M}$ exogenous flavins, FAD (F-6625, Sigma, St. Louis, MO, USA) or flavin mononucleotide (FMN) (F-8399, Sigma). The assembling efficiencies for $6 \mathrm{His}-\mathrm{F} 2$ were low when refolding buffers with $\mathrm{pH}$ lower than 7 were used. Protein-refolding of $6 \mathrm{His}-\mathrm{F} 1$ was performed using the refolding buffer containing $20 \mu \mathrm{M}$ FAD.

The refoldings of denatured $6 \mathrm{His}-\mathrm{F} 1$ and $6 \mathrm{His}-\mathrm{F} 2$ were completed by stabilization at $c a .24{ }^{\circ} \mathrm{C}$ for $20 \mathrm{~min}$ in darkness. After the insoluble proteins were removed from the refolded protein solutions by centrifugation at $10000 \mathrm{~g}$ for $30 \mathrm{~min}$ at $4{ }^{\circ} \mathrm{C}$, purification of $6 \mathrm{His}-\mathrm{F} 1$ and $6 \mathrm{His}-\mathrm{F} 2$ was performed using His-tag-binding resin (Talon metal affinity resin, BD Biosciences Clontech, Palo Alto, CA, USA) and elution buffer as described by the manufacturer.

\section{Expression and purification of 6His-(EtgPAC1)F2}

The recombinant plasmid pET21d(28a)-(EtgPAC1)F2 was introduced into E. coli strain BL21 trxB (DE3) (EMD Biosciences). The cells were cultured in LB containing $100 \mu \mathrm{g} \mathrm{ml}^{-1}$ ampicillin and $25 \mu \mathrm{g} \mathrm{ml}^{-1}$ kanamycin. 6His-(EtgPAC1)F2 was overexpressed by induction with $10 \mu \mathrm{M}$ IPTG at $15^{\circ} \mathrm{C}$ for $15 \mathrm{~h}$. The soluble 6 His-(EtgPAC1)F2 was extracted from the cells by ultrasonication and centrifugation at $10000 \mathrm{~g}$ for $30 \mathrm{~min}$ at $4{ }^{\circ} \mathrm{C}$, and was then purified by affinity chromatography using the metal affinity resin as described by the manufacturer.

\section{F1 and F2 preparations for spectrophotometric analyses}

The eluted samples of all the above described recombinant F1 and $\mathrm{F} 2$ proteins were concentrated by ultrafiltration (Ultracel YM membranes, Millipore, MA, USA). The buffers in the concentrates were replaced with fresh phosphate buffer, $50 \mathrm{mM}$ sodium phosphate, $300 \mathrm{mM} \mathrm{NaCl}$, pH 7 (BD Biosciences Clontech) for the removal of released flavins using a gel filtration chromatography kit (Biospin 6 Chromatography Columns, BioRad, Hercules, CA, USA). Appropriate protein concentrations of these F1 and F2 samples were adjusted by dilution with phosphate buffer for various spectroscopic measurements. These procedures were performed under dim-red light. The samples were dark-adapted for more than an hour and stored for less than $24 \mathrm{~h}$ on ice in darkness until use.

\section{Biochemical analyses}

The purity of recombinant $\mathrm{F} 1$ and $\mathrm{F} 2$ proteins was estimated by SDS-PAGE. Protein assays were performed by the "standardassay" method (Bio-Rad Protein Assay, Bio-Rad) as described by the manufacturer.

Heating the purified recombinant F1 and F2 solutions at $95^{\circ} \mathrm{C}$ for 3 min released flavins from denatured proteins. After cooling, denatured proteins were removed by centrifugation at $12000 \mathrm{~g}$ for $15 \mathrm{~min}$ followed by filtration (Microcon YM3 , Millipore). The extracted flavins were examined by thinlayer chromatographic (TLC) analysis using 1-butanol/acetic acid/water (4:1:5) as solvent, a silicagel plate (Silicagel 70 Plate-Wako, Wako, Osaka, Japan) and mixtures of authentic FAD (F-6625, Sigma), FMN (F-8399, Sigma) and riboflavin (R-4500, Sigma) as markers.

The concentrations of photochemically-active flavin-binding polypeptides (holoproteins) in the recombinant F2 samples for the spectroscopic analyses were estimated based on the concentrations of flavins in the recombinant F2 samples, assuming that the holoproteins bind flavins with a stoichiometry of $1: 1$. The concentrations of flavins in the recombinant F2 samples were estimated by comparing the spectra of the samples denatured by $4.5 \mathrm{M}$ guanidine- $\mathrm{HCl}$ with those of $4.5 \mathrm{M}$ guanidine- $\mathrm{HCl}$ solutions containing either FAD $\left(\varepsilon=11.3 \times 10^{3} \mathrm{M}^{-1} \mathrm{~cm}^{-1}\right.$ at $450 \mathrm{~nm})$ or $\operatorname{FMN}\left(\varepsilon=12.5 \times 10^{3} \mathrm{M}^{-1} \mathrm{~cm}^{-1}\right.$ at $\left.\left.445 \mathrm{~nm}\right)\right)^{22,23}$ The suitability of this concentration of guanidine for complete denaturation was confirmed by complete matching of the spectra of the guanidine-denatured protein solution and the pure flavins in the presence of guanidine- $\mathrm{HCl}$.

\section{Spectrophotometric analyses}

UV and visible absorption spectra of the flavin-assembled F1 and F2 samples, in darkness or under blue-light illumination, were measured at $c a .24{ }^{\circ} \mathrm{C}$ using a photodiode array spectrophotometer (model Multispec-1500, Shimadzu, Kyoto, Japan). Irradiation with blue-light was performed at $100 \mu \mathrm{mol}$ $\mathrm{m}^{-2} \mathrm{~s}^{-1}$ using a LED (model NSPB500S, peak wavelength: $465 \mathrm{~nm}$, full width at half maximum, FWHM: $28 \mathrm{~nm}$, Nichia, Tokushima, Japan).

Spectral kinetics was examined using a dual wavelength spectrophotometer (model 557, Hitachi, Tokyo, Japan) by measuring the time evolution of absorbance changes at $502 \mathrm{~nm}$, where the maximum blue light-induced red-shift was observed. The sample $(800 \mu 1)$ in a fluorometer micro-cell (FM20, GL Sciences, Tokyo, Japan) was irradiated with monochromatic blue light (442 nm) generated by a CW HeCd laser system (model 4074S-B03, Melles Griot, Carlsbad, CA, USA) at right-angles to the measuring beam. The size of the micro-cell was $10 \mathrm{~mm}$ in the measuring beam path and $2 \mathrm{~mm}$ in inside width for irradiation. To irradiate the whole sample, the laser beam was expanded by a concave lens $(20 \mathrm{~mm}$ diameter and $40 \mathrm{~mm}$ focal length, Sakai Glass, Tokyo, Japan), mounted at the outlet of the laser beam. Maximum photon fluence rate measured by a photon 
density meter (PFDM-200LX, Rayon, Tokyo, Japan) with a silicon photodiode (model S1337-66BQ, Hamamatsu Photonics, Hamamatsu, Japan) at the position of the sample (520 $\mathrm{mm}$ from the outlet for laser beam) was $c a .2 \mathrm{mmol} \mathrm{m} \mathrm{m}^{-2} \mathrm{~s}^{-1}$. Neutral density filters (Fujitok, Tokyo, Japan) were used to adjust the photon fluence rate. The actinic irradiation was turned on or off by a shutter system (model \#0, Copal, Tokyo, Japan) with an electromagnetic controller (Sigma Koki, Tokyo, Japan). Fitting curves were calculated based on measured time evolutions of absorbance changes by the method of least squares using data analysis software (Delta Graph 5.5J, Rockware, Golden, CO, USA).

\section{Results}

\section{Flavin composition}

Purity of the GST-F2 and GST-cleaved F2 samples was examined by SDS-PAGE (Fig. 2A). Only a small fraction of the expressed GST-F2 (lane 2) was soluble (lane 3). The eluates of GST-F2 (lane 4) and GST-cleaved F2 (lane 5) exhibited single bands, indicating high purity. The flavins bound to the purified GST-cleaved F2 were FAD and FMN with trace amount of riboflavin, as analysed by TLC analysis (Fig. 2B). $4.4 \mu \mathrm{M}$ GSTcleaved $\mathrm{F} 2$ contained $3.6 \mu \mathrm{M}$ mixture of flavins. The estimated amount of flavin bound to GST-cleaved F2 was $0.8 \mathrm{~mol} / \mathrm{mol}$ protein.

The insoluble $6 \mathrm{His}-\mathrm{F} 2$ was refolded into a soluble form and became the major component of the eluates, obtained by affinity chromatography with the metal affinity resin. TLC analysis identified the extracted flavin from each of these eluates to be the same as the flavin contained in each refolding-buffer used (Fig. 2B), suggesting that 6His-F2 binds exogenously added flavins. These FAD- and FMN-binding His-tagged F2 proteins were designated as $6 \mathrm{His}-\mathrm{F} 2-\mathrm{FAD}$ and $6 \mathrm{His}-\mathrm{F} 2-\mathrm{FMN}$, respectively. The purity of the $6 \mathrm{His}-\mathrm{F} 2$ at different times of overexpression and affinity purification after assembly was also examined by SDS-PAGE as shown in Fig. 2C. The eluate from the metal affinity resin also exhibited a single band, indicating sufficiently high purity. Samples of $3.9 \mu \mathrm{M} 6 \mathrm{His}-\mathrm{F} 2-$ FAD and 6 His-F2-FMN contained $3.4 \mu \mathrm{M}$ of FAD and $3.3 \mu \mathrm{M}$ of FMN, respectively. The protein solutions of 6His-F2-FAD and 6 His-F2-FMN contained flavin moieties at ratios 0.87 and $0.85 \mathrm{~mol} / \mathrm{mol}$ protein, respectively.

As for PACa F1 samples, GST-F1 exhibited a single band (Fig. 2A, lane 6), indicating high purity, and the above described procedure to recover from inclusion bodies yielded soluble 6His-F1 (Fig. 2C, lane 6). However, no flavins were detected for the PAC $\alpha$ F1 samples by TLC analysis (data not shown). This fact was confirmed by the absence of flavintype absorption in PACa F1 samples (Fig. 3) as described below.

The affinity-purified GST-F2(Y472F), GST-F2(Q514G), and 6His-(EtgPAC1)F2 also showed high purity and contained both FAD and FMN with trace amounts of riboflavin (data not shown).

\section{Absorption spectra and blue-light-induced absorbance changes of the $\mathrm{F} 1$ and $\mathrm{F} 2$ preparations}

The spectrum of dark-adapted GST-F2 (Fig. 3A) exhibited two broad absorption bands, with peaks at 381 and $454 \mathrm{~nm}$, slightly different from those of the prokaryotic BLUF domains (e.g., 365 and $445 \mathrm{~nm}$ for AppA,,${ }^{6,7} 378$ and $443 \mathrm{~nm}$ for Slr1694, ${ }^{15} 383$ and $437 \mathrm{~nm}$ for Tl10078 ${ }^{16}$ ). Dark-adapted GST-F1 did not exhibit such flavin-type broad absorption bands.

The absorption spectra of dark-adapted GST-F2(Y472F) (with peaks at $380 \mathrm{~nm}$ and $455 \mathrm{~nm}$ ), GST-F2(Q514G) (with peaks at $378 \mathrm{~nm}$ and $453 \mathrm{~nm}$ ), and 6His-(EtgPAC1)F2 (with peaks at $377 \mathrm{~nm}$ and $452 \mathrm{~nm}$ ) (Fig. 3B) were similar to those of dark-adapted GST-F2. The absorption spectrum of darkadapted GST-F2 displayed the light-induced red-shifts, but those of dark-adapted GST-F2(Y472F), GST-F2(Q514G), and 6His-(EtgPAC1)F2 did not.

Dark-adapted 6His-F2-FAD and 6His-F2-FMN showed almost the same absorption spectra as that of GST-cleaved F2 (Fig. 3C). The dark-adapted 6His-F1 did not show the flavintype two broad absorption bands (Fig. 3C).

As a consequence of the red-shift, the absorbance of GST$\mathrm{F} 2$ and GST-cleaved F2 at 394, 467, and $502 \mathrm{~nm}$ underwent significant positive changes, and that at 425,450 , and $481 \mathrm{~nm}$ significant negative changes by irradiation with blue light, but absorbance at $550 \mathrm{~nm}$ did not (Fig. 3D). The red-shifted spectrum returned in the dark to that of dark-adapted GSTcleaved F2, thus displaying a photocycle. The dark-adapted 6His-F2-FAD and 6His-F2-FMN showed almost the same redshift in the visible absorption spectra as GST-cleaved F2 (Fig. 3C and 3D). These results are similar to those of the prokaryotic BLUF domains, AppA, Slr1694, and T110078, although they display slightly different red-shifts. For example, GST-cleaved F2, 6His-F2-FAD, and 6His-F2-FMN showed the highest absorbance change at $502 \mathrm{~nm}$, whereas AppA, Slr1694, and T110078 did so at $495,{ }^{6,7} 488,{ }^{18}$ and $482^{16} \mathrm{~nm}$, respectively.

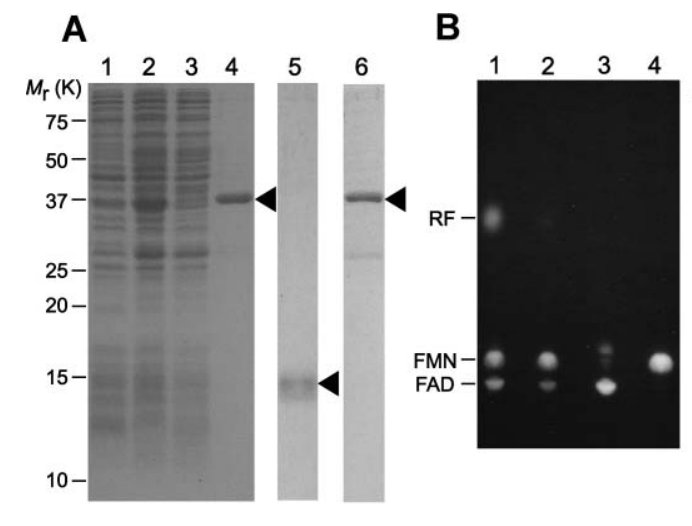

C

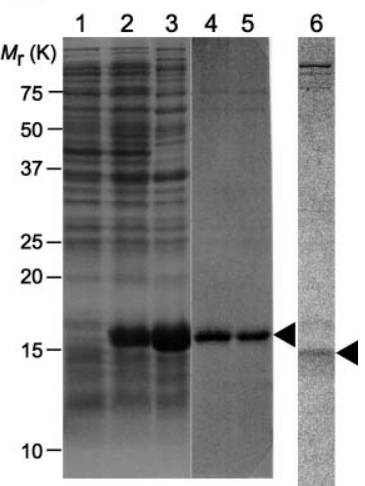

Fig. 2 Purification of recombinant PAC $\alpha$ F1 and F2 samples. (A) SDS-PAGE of overexpressed and purified GST-cleaved F2. Lane 1, crude extract of BL21 (pGEX-F2) before induction with IPTG; lane 2, BL21(pGEX-F2) 15 h after induction; lane 3, supernatant of sonicate; lane 4, affinity-purified GST-F2; lane 5, concentrate of GST-cleaved F2; lane 6, affinity-purified GST-F1. (B) TLC analysis of flavins in the recombinant PACa F2 samples. Lane 1, mixture of authentic FAD, FMN and riboflavin (RF); lane 2, GST-cleaved F2; lane 3, 6His-F2-FAD; lane 4, 6His-F2-FMN. Spots represent fluorescence emissions following illumination with UV-A. (C) SDS-PAGE of overexpressed and purified 6His-F2. Lane 1, crude extract of BL21(DE3) (pET21(28)-F2) before induction; lane 2, BL21(DE3)(pET21(28)-F2) after induction with IPTG; lane 3, inclusion body suspension; lane 4, affinity-purified 6His-F2-FAD; lane 5, affinity-purified 6His-F2-FMN; lane 6, affinity-purified 6His-F1. (A) and (C) were stained with Coomassie Brilliant Blue. 

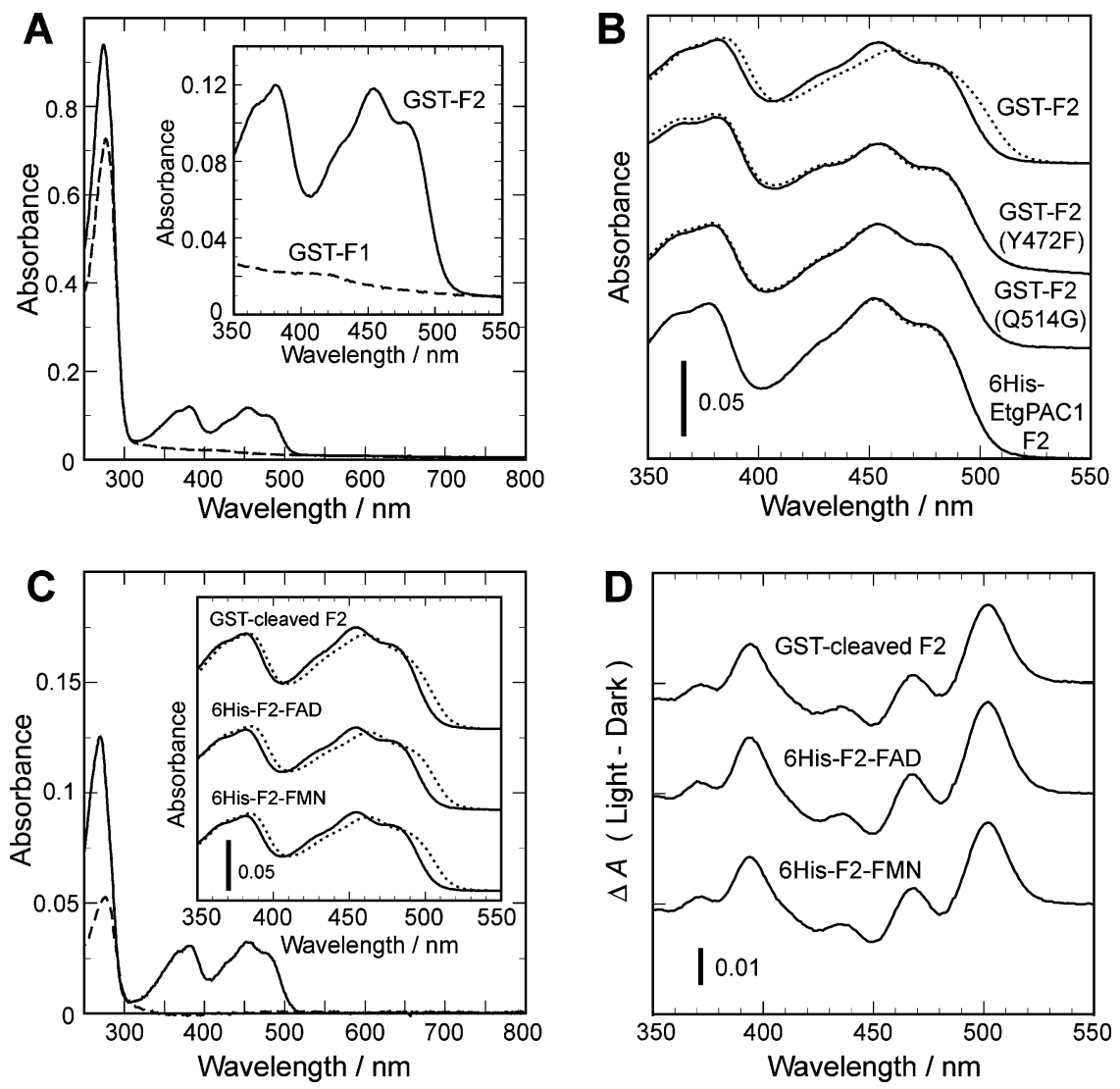

Fig. 3 UV and visible absorption (A, B, and C) and absorption difference (D) spectra of recombinant PAC $\alpha$ F1 and F2 samples. (A) The spectra of dark-adapted GST-F1 (dashed line) and GST-F2 (solid line). Inset shows the magnified spectra of dark-adapted GST-F1 and GST-F2 in the wavelength of 350 to $550 \mathrm{~nm}$. (B) The spectra of dark-adapted and blue-light-irradiated GST-F2, GST-F2(Y472F), GST-F2(Q514G), and 6His-(EtgPAC1)F2. (C) The spectra of dark-adapted 6His-F1 (dashed line) and 6His-F2-FAD (solid line). Inset shows the spectra of dark-adapted and blue-light-irradiated GST-cleaved F2, 6His-F2-FAD, and 6His-F2-FMN. (D) The absorption difference spectra of GST-cleaved F2, 6His-F2-FAD, and 6His-F2-FMN. In (B), inset of (C) and (D), the dark-adapted samples (solid line) were irradiated by a LED (465 nm, FWHM: 28 nm) at a photon fluence rate of $100 \mu \mathrm{mol} \mathrm{m} \mathrm{m}^{-2} \mathrm{~s}^{-1}$. Spectra of light-irradiated samples (dotted line) were acquired at $60 \mathrm{~s}$ after the onset of irradiation.

\section{Kinetic characterization of photocycles of different $\mathbf{F 2}$ preparations}

The time evolutions of absorbance changes at $502 \mathrm{~nm}$, designated as $\Delta A_{502 \mathrm{~nm}}$, are shown in Fig. $4 \mathrm{~A} . \Delta A_{502 \mathrm{~nm}}$ at various photon fluence rates below $100 \mu \mathrm{mol} \mathrm{m} \mathrm{m}^{-2} \mathrm{~s}^{-1}$, were measured at $25^{\circ} \mathrm{C}$ for dark-adapted GST-cleaved F2, 6His-F2-FAD, and 6His-F2FMN samples with $3.6 \mu \mathrm{M}$ flavins at estimated molar ratios of $1: 4$ (FAD : FMN) (cf. Fig. 2B), $3.4 \mu \mathrm{M} \mathrm{FAD}$, and $3.3 \mu \mathrm{M} \mathrm{FMN}$, respectively. Moreover, photocycles were examined on 6His-F2FAD and 6 His-F2-FMN at various concentrations (below $8 \mu \mathrm{M}$ flavins in these recombinant $\mathrm{F} 2$ samples).

The time resolved $\Delta A_{502 \mathrm{~nm}}$ were analyzed by assuming the following simplified photochemical model with a ground state (A) and a signaling state (B) which causes the red-shift of absorption spectrum for the light-reaction of photocycle at room temperature:

$$
\mathrm{A} \underset{k_{2}}{\stackrel{k_{1}}{\rightleftarrows}} \mathrm{B}
$$

where $k_{1}$ and $k_{2}$ are rate constants. $k_{1}$ is given by the equation $k_{1}=\Phi \kappa E_{\mathrm{p}, \mathrm{o}}$, where $\Phi$ is the quantum efficiency, $\kappa$ is the molar absorption cross section and $E_{\mathrm{p}, \mathrm{o}}$ is the photon fluence rate. In the dark-relaxation phase the formation of B stops, and $\mathrm{B}$ returns to A with the rate constant $k_{2}$. This photochemical reaction model gives the simultaneous differential equations for the concentrations of $\mathrm{A}$ and $\mathrm{B}, \mathrm{d}[\mathrm{A}] / \mathrm{d} t=-k_{1}[\mathrm{~A}]+k_{2}[\mathrm{~B}]$ and $\mathrm{d}[\mathrm{B}] / \mathrm{d} t=k_{1}[\mathrm{~A}]-k_{2}[\mathrm{~B}]$. By substituting solutions of these differential equations into equation $\Delta A_{502 \mathrm{~nm}}=\varepsilon_{\mathrm{A}}[\mathrm{A}]+\varepsilon_{\mathrm{B}}[\mathrm{B}]-$
$\varepsilon_{\mathrm{A}}[\mathrm{A}]_{0}$, the calculated time-dependent values of $\Delta A_{502 \mathrm{~nm}}$ are given by the following equations,

$$
\begin{gathered}
\Delta A_{502 \mathrm{~nm}} \text { (light-on) }=\Delta A_{\max }\left(1-\mathrm{e}^{-k_{\mathrm{on} t} t}\right) \\
\Delta A_{502 \mathrm{~nm}} \text { (light-off) }=\Delta A_{\max } \mathrm{e}^{-k_{\text {off }} t}
\end{gathered}
$$

where the values of $t$ in equations (2) and (3) are the blue-light irradiation time and the decaying time in darkness, respectively. $[\mathrm{A}]_{0}$ is the initial concentration of flavin-binding protein (A), and given by equation $[\mathrm{A}]_{0}=[\mathrm{A}]+[\mathrm{B}] . \varepsilon_{\mathrm{A}}$ and $\varepsilon_{\mathrm{B}}$ are molar absorption coefficients at $502 \mathrm{~nm}$ for $\mathrm{A}$ and $\mathrm{B}$, respectively. $\Delta A_{\max }$ is the saturated (maximum) level in a stationary state of $\Delta A_{502 \mathrm{~nm}}$, $k_{\text {on }}$ is the observed rate constant of light-reaction, and $k_{\text {off }}$ is the observed rate constant of dark-relaxation. The initial velocity $\left(V_{0}\right)$ is a slope of a tangent line to the time evolution curve of $\Delta A_{502 \mathrm{~nm}}$ at the onset of irradiation and given by equation $V_{0}=$ $\Delta A_{\max } k_{\text {on }} \cdot \Delta A_{\max }, k_{\text {on }}, V_{0}$, and $k_{\text {off }}$ are related to the photon fluence rate $\left(E_{\mathrm{p}, \mathrm{o}}\right)$ by the following equations,

$$
\begin{gathered}
\Delta A_{\max }=\frac{\left(\varepsilon_{\mathrm{B}}-\varepsilon_{\mathrm{A}}\right) \Phi \kappa E_{\mathrm{p}, \mathrm{o}}[\mathrm{A}]_{0}}{\Phi \kappa E_{\mathrm{p}, \mathrm{o}}+k_{2}} \\
k_{\mathrm{on}}=\Phi \kappa E_{\mathrm{p}, \mathrm{o}}+k_{2} \\
V_{0}=\left(\varepsilon_{\mathrm{B}}-\varepsilon_{\mathrm{A}}\right) \Phi \kappa E_{\mathrm{p}, \mathrm{o}}[\mathrm{A}]_{0} \\
k_{\mathrm{off}}=k_{2}
\end{gathered}
$$

Calculated and measured values of $\Delta A_{502 \mathrm{~nm}}$ were in good agreement for all the samples of GST-cleaved F2, 6His-F2FAD and 6His-F2-FMN (Fig. 4). Fig. 5 shows that these kinetic 

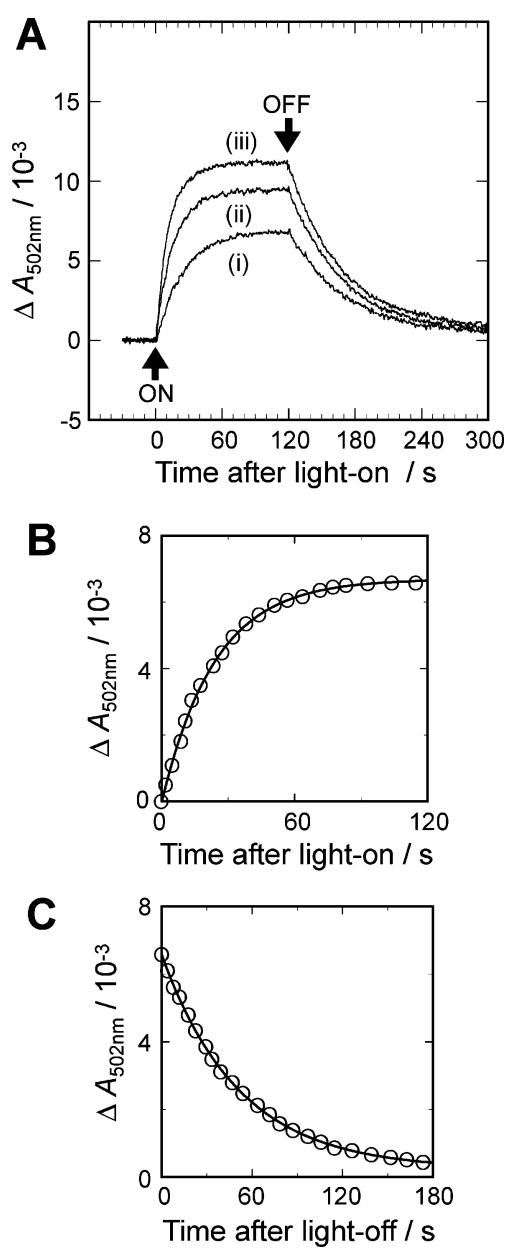

Fig. 4 Light-induced absorption changes of GST-cleaved F2. (A) The time evolutions of $\Delta A_{502 \mathrm{~nm}}$ were measured at $25^{\circ} \mathrm{C}$ using a dual wavelength spectrophotometer under irradiation with $442 \mathrm{~nm}$ (generated by a $\mathrm{HeCd}$ laser system) at photon fluence rates of 24 (i), 47 (ii), and 90 (iii) $\mu \mathrm{mol} \mathrm{m}{ }^{-2} \mathrm{~s}^{-1}$, respectively, for GST-cleaved F2, which contained $3.6 \mu \mathrm{M}$ flavins at an estimated molar ratio of $1: 4$ (FAD : FMN). Arrows indicate timings of turning on and off of irradiation. (B) Calculated curve and plots (circles) of raw data for the photoinduced reaction (i). (C) Calculated curve and plots (circles) of raw data for dark-relaxation on measurement (i).

characteristics of $\Delta A_{\max }, k_{\mathrm{on}}, V_{0}$ and $k_{\mathrm{off}}$ were in good agreement with eqn (4) to (7), respectively, relating with $E_{\mathrm{p}, \mathrm{o}}$ and $[\mathrm{A}]_{0}$. These experimental results for recombinant PAC $\alpha$ F2 support the validity of the simplified photochemical model (1).

From the plots of $k_{\text {off }}$ (Fig. 5D), the values of half-life in darkrelaxation, given by equation $t_{1 / 2}$ off $=\ln 2 k_{\mathrm{off}}{ }^{-1}$, for GST-cleaved F2, 6His-F2-FAD, and 6His-F2-FMN were determined to be 34,44 , and $40 \mathrm{~s}$, respectively. From the plots of $k_{\text {on }}$ (Fig. 5B), the values of $\Phi_{\kappa}$, were determined. The values of $\kappa$ at $442 \mathrm{~nm}$ were estimated by equation $\kappa=2.303 \varepsilon_{442}$, where $\varepsilon_{442}$ is the molar absorption coefficient at $442 \mathrm{~nm}$ for dark-adapted samples. The values of $\varepsilon_{442}$ for GST-cleaved F2, 6His-F2-FAD, and 6His-F2FMN were $10.9 \times 10^{3}, 10.4 \times 10^{3}$, and $10.8 \times 10^{3} \mathrm{M}^{-1} \mathrm{~cm}^{-1}$, respectively. With these values, the values of $\Phi$ for GST-cleaved F2, 6His-F2-FAD, and 6His-F2-FMN were calculated to be $0.32,0.28$, and 0.28 , respectively. These values are similar to those for AppA $(0.24)^{13}$ and Tllo078 (0.29). ${ }^{16}$ From the plots of $\Delta A_{\max }$ shown in Fig. $5 \mathrm{~A}$, or those of $V_{0}$ shown in Fig. $5 \mathrm{C}$, the values of $\left(\varepsilon_{\mathrm{B}}-\varepsilon_{\mathrm{A}}\right)[\mathrm{A}]_{0}$ were determined. The estimated values of $\varepsilon_{\mathrm{A}}$ were $2.0 \times 10^{3}, 2.1 \times 10^{3}$, and $2.1 \times 10^{3} \mathrm{M}^{-1} \mathrm{~cm}^{-1}$ for the dark-adapted GST-cleaved F2, 6His-F2-FAD, and 6His-F2FMN. With these values, $\varepsilon_{\mathrm{B}}$ for GST-cleaved F2, 6His-F2-FAD, and 6 His-F2-FMN were calculated to be $6.0 \times 10^{3}, 5.7 \times 10^{3}$, and $5.7 \times 10^{3} \mathrm{M}^{-1} \mathrm{~cm}^{-1}$, respectively.

\section{Discussion}

\section{Selectivity in flavin-binding by $F 2$ and prokaryotic BLUF domains}

Purified GST-cleaved F2 bound not only FAD but also FMN and riboflavin although native PAC seems to bind only FAD. ${ }^{1,2}$ At first glance, this may indicate that extracted FMN was a decomposition product of FAD initially bound to recombinant F2 during expression and/or purification. Taken together, however, the facts that the ultracentrifuged supernatant of boiled homogenate of the cell contained FAD and FMN (data not shown) and that 6His-F2 could bind either FAD or FMN by assembly as described above, it is more probable that the extracted FMN was bound to recombinant F2 from the beginning of expression in E. coli.

Thus, recombinant F2 can bind either FAD or FMN. A possible interpretation for the exclusive FAD binding of the native PAC protein might be that only native PAC with FAD could fold into the correct (e.g., the $\alpha_{2} \beta_{2}$ heterotetramer) protein structure.

The spectral characteristics of the assembled $6 \mathrm{His}-\mathrm{F} 2$ binding FAD and 6His-F2 binding FMN were similar to those of GST-cleaved F2. The rate constants of light-reaction and darkrelaxation of the 6 His-F2-FAD and 6 His-F2-FMN are slightly lower than those of GST-cleaved F2.

The kinetic characteristics of photocycles of 6His-F2-FAD and 6 His-F2-FMN were nearly the same. This fact suggests that the adenine ring of FAD is not essential in the photochemical reaction of the photocycle, at least in recombinant PAC $\alpha$ F2. The rate constants of light-reaction and dark-relaxation of the former are just slightly smaller than those of the latter. Similarly, it was found that the rate constants of the lightreaction and dark-relaxation of the assembled BLUF domain of AppA binding FAD are smaller than those of the one binding FMN. ${ }^{11}$ Such slight differences in the kinetic characteristics of the photocycle between FAD-binding and FMN-binding BLUF domains should then result from some factor other than the isoalloxazine ring (e.g., the adenine ring).

\section{Differences in the two BLUF domains, F1 and F2, of PAC}

The interchanged F1 in place of the BLUF domain of AppA mediated light-dependent gene expression in $R$. sphaeroides. ${ }^{24}$ This fact suggests that the mechanisms of photosensing of eukaryotic and prokaryotic BLUF domains are essentially the same even though they are involved in different types of photoreactions, i.e. photomovements through activation of the adenylyl cyclase domain in the former and gene expression or photomovement control in the latter. This report also indicates that F1 can play a role as a blue-light sensing domain in vivo at least when heterologously expressed in $R$. sphaeroides. It is assumed that PAC $\alpha$ F1 binds FAD, because PAC $\alpha$ F1 and $\mathrm{PAC} \alpha \mathrm{F} 2$ have similar homologous regions. Strangely enough, however, recombinant PAC $\alpha \mathrm{F} 1$ samples (the region from Gly48 to His158 of PAC $\alpha$ ) did not bind flavins (Fig. 3A and 3C), although we could obtain sufficient amount of the soluble recombinant PAC $\alpha \mathrm{F} 1$ by heterologous expression with fused GST in E. coli (BL21) and the above described procedure to recover from inclusion bodies gave the soluble recombinant PAC $\alpha$ F1 (6His-F1). The reason why no flavins bind to the recombinant $\mathrm{PAC} \alpha \mathrm{F} 1$ samples is not yet clear.

It is noteworthy that in phototropin, another flavin-based blue-light sensor that contains two flavin-binding domains (LOV1 and LOV2), phot1 LOV2 exhibits a higher photosensitivity and plays a more important role in the physiological response than LOV1 in phot $1 .{ }^{25}$ The quantum efficiencies of phot1 LOV2 are 10-fold higher [0.44 (oat), 0.34 (Arabidopsis), 0.30 (rice)] than those of phot1 LOV1 [0.045 (oat), 0.035 (Arabidopsis), 0.026 (rice)] of higher plants. ${ }^{26,27}$ A recent report suggests an interaction between Chlamydomonas reinhardtii phototropin 

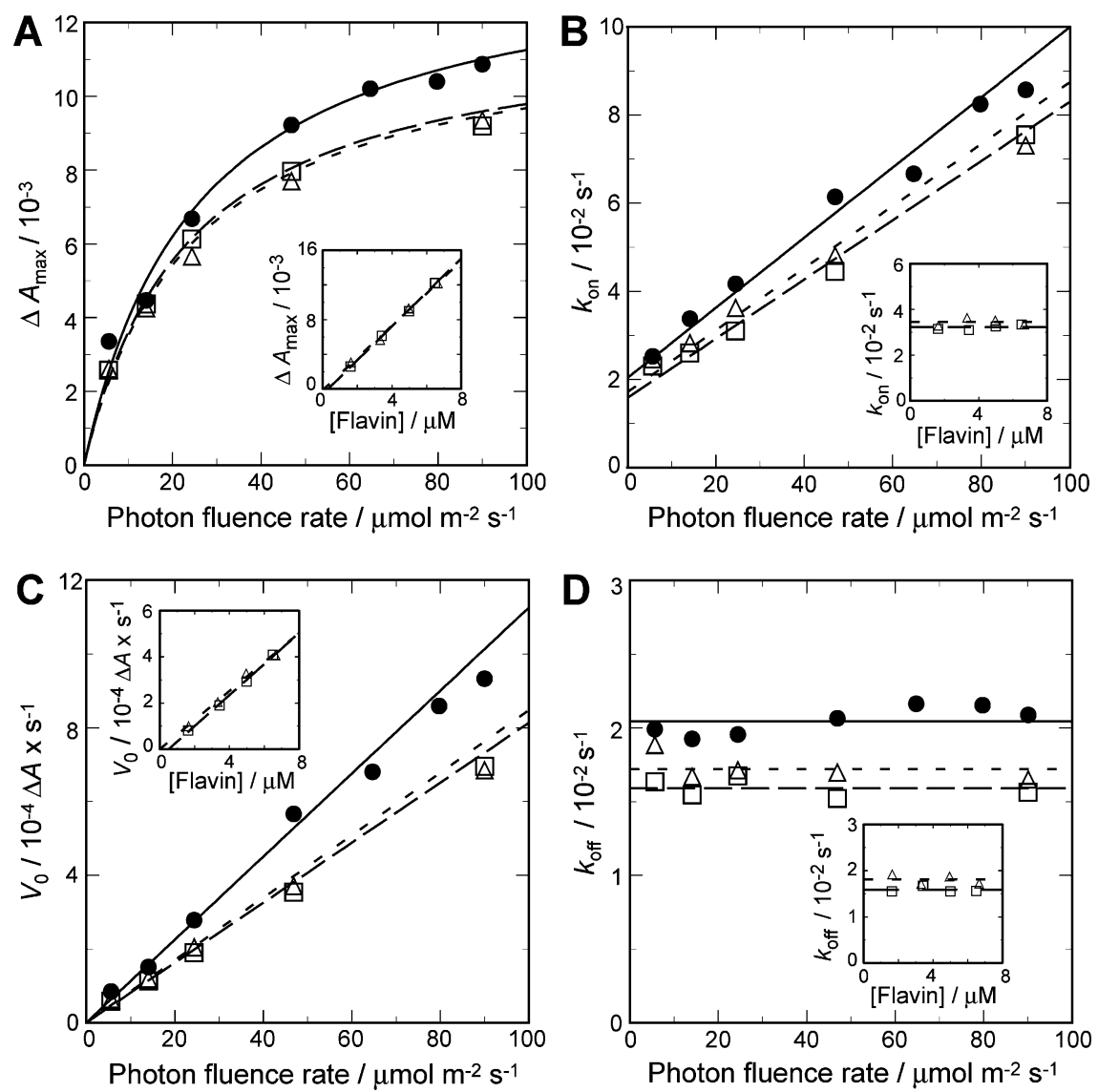

Fig. 5 Kinetic characteristics of photocycle versus photon fluence rate for GST-cleaved F2 (- - ) , 6His-F2-FAD (- $\square-$ ), and 6His-F2-FMN (--- $\triangle$---). (A) Maximum $\Delta A_{502 \mathrm{~nm}}\left(\Delta A_{\max }\right)$. (B) Observed rate constant $\left(k_{\mathrm{on}}\right)$ upon irradiation. (C) Initial velocities $\left(V_{0}\right)$. (D) Observed rate constant $\left(k_{\text {off }}\right)$ during dark-relaxation. Kinetic values were determined by exponential curve fitting of the measured time evolutions of $\Delta A_{502} \mathrm{~nm}$ at $25^{\circ} \mathrm{C}$ upon irradiation at $442 \mathrm{~nm}$, at various photon fluence rates below $100 \mu \mathrm{mol} \mathrm{m}{ }^{-2} \mathrm{~s}^{-1}$ for dark-adapted GST-cleaved F2, 6His-F2-FAD, and 6His-F2-FMN samples, with $3.6 \mu \mathrm{M}$ flavins with estimated molar ratios of $1: 4$ (FAD : FMN), $3.4 \mu \mathrm{M}$ FAD, and $3.3 \mu \mathrm{M}$ FMN, respectively. Kinetic values in insets were determined for samples with various protein concentrations for 6His-F2-FAD and 6His-F2-FMN. The horizontal axes of the insets show the concentration of flavins in recombinant F2 samples. Irradiation was performed at $24 \mu \mathrm{mol} \mathrm{m}^{-2} \mathrm{~s}^{-1}$.

LOV1 and LOV2 domains in the photocycle, indicating that decay behavior of photoproducts in the coexisting domains is different from that in individual domains. ${ }^{28}$ In this context, the photocycle characteristics of F1 and F2 should be compared both separately and jointly. Because of this, it is not clear which domains of F1 and F2 play more important roles in photoactivation of PAC.

\section{Comparison of photocycling characteristics of $\mathrm{F} 2$ preparations with those of prokaryotic BLUF domains}

The recombinant Y472F and Q514G mutants of PAC $\alpha$ F2 did not show a light-induced red-shift of absorption (Fig. 3B), similarly to the Y21F mutant of AppA and the Y8F mutant of Slr1694, and Q50A of Tl10078. Recombinant EtgPAC1 F2, although having the tyrosine residue, did not show the spectral red-shift (Fig. 3B). These facts indicate that the tyrosine residue and the glutamine residue conserved in PAC play essential roles in the photocycle in recombinant PAC $\alpha$ F2, similarly to prokaryotic BLUF domains.

As described above, the eukaryotic BLUF domain of PAC showed essentially the same photocycle as prokaryotic BLUF domains in the time windows studied, from milliseconds to minutes. The values of $t_{1 / 2}$ off of $34-44 \mathrm{~s}$ at $25^{\circ} \mathrm{C}$ for recombinant $\mathrm{PAC} \alpha \mathrm{F} 2$ is closer to that of cyanobacterial BLUF domains [Slr1694 $(\sim 11 \mathrm{~s})^{14}$ and T110078 $\left.(\sim 3.5 \mathrm{~s})^{14}\right]$, rather than that of the purple bacterial BLUF domain [AppA $\left.(900 \mathrm{~s})^{6,7}\right]$. Taking into account that not only PAC but also Slr1694 and T110078 probably mediate photomovements, their relatively short recovery times may be needed for maintenance of the photomovement responses in contrast to the gene expression control mediated by the latter (AppA), which is a relatively slow response. The recently reported rapid formation of the signaling state (within $10 \mathrm{~ns})^{13,16}$ in contrast with the longer and diverse lifetime (i.e. $t_{1 / 2}$ off of several seconds to $900 \mathrm{~s}$ ) of prokaryotic BLUF domains, raises the question about the mechanism by which the diversity of time parameters are caused. In this regard it is noteworthy that an extracted PAC solution responded to repetitive blue light pulses of various durations (down to $50 \mathrm{~ms}$ ) with equal efficiencies. ${ }^{29}$ The molecular mechanism of putative interaction between the BLUF domain(s) and the adenylyl cyclase domains in the photoactivation of PAC constitutes a challenging subject for future studies.

\section{Acknowledgements}

We are grateful to Dr Shinji Masuda and $\mathrm{Mr}$ Takanori Nakamura for valuable advice, and Ms Yumiko Makino for spectroscopical support. We acknowledge Drs Chris Bowler and Silvia Braslavsky for reading the manuscript. This work was supported in part by a Grant-in-Aid for Scientific Research from the Ministry of Education, Science, Sports and Culture of Japan (15207006 to MW and 17657018 to AM) and the Foundation for Promotion of Photosciences and Phototechnologies. S. Ito acknowledges National Institute for Basic Biology, for a fellowship during part of this study.

\section{References}

1 M. Iseki, S. Matsunaga, A. Murakami, K. Ohno, K. Shiga, K. Yoshida, M. Sugai, T. Takahashi, T. Hori and M. Watanabe, A bluelight-activated adenylyl cyclase mediates photoavoidance in Euglena gracilis, Nature, 2002, 415, 1047-1051. 
2 M. Watanabe and M. Iseki, Discovery and characterization of photoactivated adenylyl cyclase (PAC), a novel blue-light receptor flavoprotein, from Euglena gracilis in Handbook of Photosensory Receptors, ed. W. R. Briggs and J. L. Spudich, Wiley-VCH Verlag GmbH \& Co. KGaA, Weinheim, 2005, pp. 447-460.

$3 \mathrm{M}$. Gomelsky and G. Klug, BLUF: a novel FAD-binding domain involved in sensory transduction in microorganisms, Trends Biochem. Sci., 2002, 27, 497-500.

4 Y. Koumura, T. Suzuki, S. Yoshikawa, M. Watanabe and M. Iseki, The origin of photoactivated adenylyl cyclase (PAC), the Euglena blue-light receptor: phylogenetic analysis of orthologues of PAC subunits from several euglenoids and trypanosome-type adenylyl cyclase from Euglena gracilis, Photochem. Photobiol. Sci., 2004, 3, 580-586.

5 M. Gomelsky and S. Kaplan, AppA, a redox regulator of photosystem formation in Rhodobacter sphaeroides 2.4.1, is a flavoprotein, J. Biol. Chem., 1998, 52, 35319-35325.

6 S. Masuda and C. E. Bauer, AppA is a blue light photoreceptor that antirepresses photosynthesis gene expression in Rhodobacter sphaeroides, Cell, 2002, 110, 613-623.

7 B. J. Kraft, S. Masuda, J. Kikuchi, V. Dragnea, G. Tollin, J. M. Zaleski and Carl E. Bauer, Spectroscopic and mutational analysis of the blue-light photoreceptor AppA: A novel photocycle involving flavin stacking with an aromatic amino acid, Biochemistry, 2003, 42, 6726-6734.

8 S. Masuda and C. E. Bauer, The antirepressor AppA uses the novel flavin-binding BLUF domain as a blue-light-absorbing photoreceptor to control photosystem synthesis in Handbook of Photosensory Receptors, ed. W. R. Briggs and J. L. Spudich, Wiley-VCH Verlag GmbH \& Co. KGaA, Weinheim, 2005, pp. 433-445.

9 W. Laan, M. A. van der Horst, I. H. van Stokkum and K. J. Hellingwerf, Initial characterization of the primary photochemistry of AppA, a blue-light-using flavin adenine dinucleotide-domain containing transcriptional antirepressor protein from Rhodobacter sphaeroides: a key role for reversible intramolecular proton transfer from the flavin adenine dinucleotide chromophore to a conserved tyrosine?, Photochem. Photobiol., 2003, 78, 290-297.

10 M. A. van der Horst and K. J. Helligwerf, Photoreceptor proteins, "star actors of modern times": a review of the functional dynamics in the structure of representative members of six different photoreceptor families, Acc. Chem. Res., 2004, 37, 13-20.

11 W. Laan, T. Bednarz, J. Heberle and K. J. Hellingwerf, Chromophore composition of a heterologously expressed BLUF-domain, Photochem. Photobiol. Sci., 2004, 3, 1011-1016.

$12 \mathrm{~S}$. Masuda, K. Hasegawa and T. Ono, Light-induced structural changes of apoprotein and chromophore in the sensor of blue light using FAD (BLUF) domain of AppA for a signaling state, Biochemistry, 2005, 44, 1215-1224.

13 M. Gauden, S. Yeremenko, W. Laan, I. H. M. van Stokkum, J. A. Ihalainen, R. van Grondelle, K. J. Hellingwerf and J. T. M. Kennis, Photocycle of the flavin-binding photoreceptor AppA, a bacterial transcriptional antirepressor of photosynthesis genes, Biochemistry, 2005, 44, 3653-3662.

14 K. Okajima, S. Yoshihara, Y. Fukushima, X. Geng, M. Katayama, S. I. Higashi, M. Watanabe, S. Sato, S. Tabata, Y. Shibata, S. Itoh and M. Ikeuchi, Biochemical and functional characterization of BLUF-type flavin-binding proteins of two species of cyanobacteria, J. Biochem., 2005, 137, 741-750.
15 S. Masuda, K. Hasegawa, A. Ishii and T. Ono, Light-Induced structual changes in a putative blue-light receptor with a novel FAD binding fold sensor of blue-light using FAD (BLUF); Slr1694 of Synechocystis sp. PCC6803, Biochemistry, 2004, 43, 5304-5313.

16 Y. Fukushima, K. Okajima, Y. Shibata, M. Ikeuchi and S. Itoh, Primary intermediate in the photocycle of a blue-light sensory BLUF FAD-protain, T110078, of Thermosynechococcus elongatus BP1, Biochemistry, 2005, 44, 5149-5158.

17 K. Hasegawa, S. Masuda and T. Ono, Spectroscopic analysis of the dark relaxation process of a photocycle in a sensor of blue light using FAD (BLUF) protein Slr1694 of the cyanobacterium Synechocystis sp. PCC6803, Plant Cell Physiol., 2005, 46, 136-146.

18 K. Hasegawa, S. Masuda and T. Ono, Structual intermediate in the photocycle of BLUF (sensor of blue light using FAD) protein Slr1694 in a cyanobacterium Synechocystis sp. PCC6803, Biochemistry, 2004 43, 14979-14986.

19 A. Kita, K. Okajima, Y. Morimoto, M. Ikeuchi and K. Miki, Structure of a Cyanobacterial BLUF Protein, Tl10078, Containing a novel FAD-binding blue light sensor domain, J. Mol. Biol., 2005, 349, $1-9$.

20 S. Anderson, V. Dragnea, S. Masuda, J. Ybe, K. Moffat and C. Bauer, Structure of a novel photoreceptor, the BLUF domain of AppA from Rhodobacter sphaeroides, Biochemistry, 2005, 44, 7998-8005.

21 M. Watanabe, M. Iseki, S. Ito and A. Murakami, in: Abstracts to the 14th International Congress on Photobiology, June 10 to 15, Jeju, Korea, 2004, abstract S12-07, P. 107.

22 C. Walsh, J. Fisher, R. Spencer, D. W. Graham, W. T. Ashton, J. E. Brown, R. D. Brown and E. F. Rogers, Chemical and enzymatic properties of riboflavin analogues, Biochemistry, 1978, 17, 19421951.

23 C. Thorpe, R. G. Mathews and C. H. Williams, Acyl-coenzyme A dehydrogenase from pig kidney: purification and properties, Biochemistry, 1979, 18, 331-337.

24 Y. Han, S. Braatsch, L. Osterloh and G. Klug, A eukaryotic BLUF domain mediates light-dependent gene expression in the purple bacterium Rhodobacter sphaeroides 2.4.1, Proc. Natl. Acad. Sci. USA, 2004, 101, 12306-12311.

25 J. M. Christie and W. R. Briggs, Blue light sensing and signaling by the phototropins in Handbook of Photosensory Receptors, ed. W. R. Briggs and J. L. Spudich, Wiley-VCH Verlag GmbH \& Co. KGaA, Weinheim, 2005, pp. 277-303.

26 M. Salomon, J. M. Christie, E. Knieb, U. Lempert and W. R. Briggs, Photochemical and mutational analysis of the FMN-binding domains of the plant blue light receptor, phototropin, Biochemistry, 2000, 39, 9401-9410.

27 M. Kasahara, T. E. Swartz, M. A. Olney, A. Onodera, N. Mochizuki, H. Fukuzawa, E. Asamizu, S. Tabata, H. Kanegae, M. Takano, J. M. Christie, A. Nagatani and W. R. Briggs, Photochemical properties of the flavin mononucleotide-binding domains of the phototropins from Arabidopsis, rice, and Chlamydomonas reinhardtii, Plant Physiol., 2002, 129, 762-773.

28 H. Guo, T. Kottke, P. Hegemann and B. Dick, The Phot LOV2 domain and its interaction with LOV1, Biophys J., 2005, 89, 402412.

29 S. Yoshikawa, T. Suzuki, M. Watanabe and M. Iseki, Kinetic analysis of the activation of photoactivated adenylyl cyclase (PAC), a blue-light receptor for photomovements of Euglena, Photochem. Photobiol. Sci., 2005, DOI: 10.1039/b417212d. 